\title{
Chromosomally normal miscarriage is associated with vaginal dysbiosis and local inflammation
}

\author{
Karen Grewal ${ }^{1 *}$ (D, Yun S. Lee ${ }^{1,2}$, Ann Smith ${ }^{3}$, Jan J. Brosens ${ }^{4}$, Tom Bourne ${ }^{1}$, Maya Al-Memar ${ }^{1}$, Samit Kundu²,
} David A. Maclntyre ${ }^{1,2}$ and Phillip R. Bennett ${ }^{1,2}$

\begin{abstract}
Background: Emerging evidence supports an association between vaginal microbiota composition and risk of miscarriage; however, the underlying mechanisms are poorly understood. We aim to investigate the vaginal microbial composition and the local immune response in chromosomally normal and abnormal miscarriages and compare this to uncomplicated pregnancies delivering at term.
\end{abstract}

Methods: We used 16S rRNA gene based metataxonomics to interrogate the vaginal microbiota in a cohort of 167 women, 93 miscarriages (54 euploid and 39 aneuploid using molecular cytogenetics) and 74 women who delivered at term and correlate this with the aneuploidy status of the miscarriages. We also measured the concentrations of $\mathrm{IL}-2, \mathrm{IL}-4, \mathrm{IL}-6, \mathrm{IL}-8, \mathrm{TNF}-\mathrm{a}, \mathrm{IFN}-\gamma, \mathrm{IL}-1 \beta, \mathrm{IL}-18$ and IL-10 in cervical vaginal fluid.

Results: We show that euploid miscarriage is associated with a significantly higher prevalence of Lactobacillus spp. deplete vaginal microbial communities compared to aneuploid miscarriage $(P=0.01)$. Integration of matched cervicovaginal fluid immune-profiles showed that Lactobacillus spp. depleted vaginal microbiota associated with pro-inflammatory cytokine levels most strongly in euploid miscarriage compared to viable term pregnancy (IL-1 $\beta ; P$ $<0.001, \mathrm{IL}-8 ; P=0.01, \mathrm{IL}-6 ; P<0.001)$.

Conclusions: Our data suggest the vaginal microbiota plays an important aetiological role in euploid miscarriage and may represent a target to modify risk of pregnancy loss.

Keywords: Miscarriage, Microbiota, Infectious disease, Early pregnancy, Translational research

\section{Background}

Miscarriage, defined as pregnancy loss before the fetus reaches viability, is a distressing disorder associated with pain and bleeding as well as significant psychological morbidity. Early miscarriage (pregnancy loss before 12 weeks) occurs in one in five pregnancies of which half are due to chromosomal abnormalities [1]. Infection is

\footnotetext{
* Correspondence: karen.grewal04@imperial.ac.uk

'Tommy's National Centre for Miscarriage Research, Institute of Reproductive \& Developmental Biology, Imperial College London, Hammersmith Hospital Campus, Du Cane Road, London, UK

Full list of author information is available at the end of the article
}

implicated in $66 \%$ of late miscarriages (12-24 weeks) but is less prevalent in early miscarriage [2]. However, the mechanisms driving miscarriage in these groups are poorly defined. Despite its prevalence, there are no interventions that prevent sporadic miscarriage and only few treatments, such as progesterone supplements, have been shown to modestly reduce the recurrence risk of miscarriage in subsequent pregnancies [3].

Pregnancy has a unique and dynamic immunological milieu that is required to support a healthy pregnancy [4]. Initially, a pro-inflammatory state is required for implantation which involves a release of inflammatory

(C) The Author(s). 2022 Open Access This article is licensed under a Creative Commons Attribution 4.0 International License, which permits use, sharing, adaptation, distribution and reproduction in any medium or format, as long as you give appropriate credit to the original author(s) and the source, provide a link to the Creative Commons licence, and indicate if changes were made. The images or other third party material in this article are included in the article's Creative Commons licence, unless indicated otherwise in a credit line to the material. If material is not included in the article's Creative Commons licence and your intended use is not permitted by statutory regulation or exceeds the permitted use, you will need to obtain permission directly from the copyright holder. To view a copy of this licence, visit http://creativecommons.org/licenses/by/4.0/ The Creative Commons Public Domain Dedication waiver (http://creativecommons.org/publicdomain/zero/1.0/) applies to the data made available in this article, unless otherwise stated in a credit line to the data. 
mediators inducing tissue injury and repair [5]. Infection may disrupt the immunological synergy at implantation and trigger adverse outcome [6]. For example, chlamydial infection has been shown to cause dysregulation of decidualisation [7], which can contribute to miscarriage by impairing implantation and trophoblast invasion. As pregnancy progresses, there is broadly resolution of inflammation until close to term, a pro-inflammatory state then returns and contributes to the mechanisms of the onset of labour [8]. However, a continuous antiinflammatory state in pregnancy is considered over simplified and has since been disputed. Further work has described localized changes in response to infection or 'dangerous' signals [9]. Therefore, failed tolerance in certain women at the maternal fetal interface and inappropriate, premature activation of inflammation in the reproductive tract may lead to miscarriage or preterm birth [10].

Emerging evidence implicates the reproductive tract microbiota as a key modulator of local inflammatory and immune pathways throughout pregnancy. During pregnancy, increased oestrogen levels promote glycogen deposition in the vaginal epithelia, consequently supporting Lactobacillus spp. dominance and stability during pregnancy [11-13]. This relationship provides protection against pathogenic bacteria by producing lactic acid and antimicrobial compounds such as bacteriocins [14]. Depletion in vaginal Lactobacillus spp. is linked to adverse pregnancy outcomes, including preterm birth and preterm prelabour rupture of fetal membranes (PPROM) [15-17]. There is a correlation between vaginal communities deplete in Lactobacillus spp. and levels of pro-inflammatory cytokines within the cervicovaginal fluid (CVF), suggesting a mechanistic link between an aberrant vaginal microbial environment and adverse pregnancy outcome $[18,19]$.

In a recent longitudinal study of the pregnancy vaginal microbiome starting at 6 weeks of gestation, we showed that women with vaginal communities dominated by species other than Lactobacillus spp. at any point during gestation were at increased risk of PPROM [20]. We have also recently shown that a Lactobacillus spp. depleted vaginal microbiome in early pregnancy is a risk factor for first trimester miscarriage [21]. However, this study did not distinguish between euploid and aneuploid pregnancy losses, nor did it explore the relationships between the vaginal microbiota and inflammatory mediators. We hypothesise that whilst aneuploid pregnancies are lost mainly because of intrinsic developmental errors, euploid miscarriages may be caused by inflammatory signals triggered by an adverse vaginal microbiota composition. To test this hypothesis, we characterized the vaginal microbiota and host immune response in women with chromosomally normal and abnormal miscarriages as well as in uncomplicated pregnancies that successfully progressed to term.

\section{Methods \\ Study population and study design}

This study was a prospective observational cohort study based at Queen Charlotte's \& Chelsea Hospital, Early Pregnancy Unit, London between March 2014 and February 2019. The study was approved by NHS National Research Ethics Service (NRES) (REC 16/WA/0357 and REC 14/LO/0199). All participants provided written informed consent. Patients were not involved in the development of the research. Patients were recruited either when they presented initially with a confirmed miscarriage diagnosis, or when they presented with pain and/or bleeding without an initially confirmed miscarriage diagnosis in the first trimester of pregnancy. The first trimester was defined as < 14 weeks' gestation by last menstrual period (LMP) or, where LMP was not known, ultrasound scan dating based on crown-rump length measurements (CRL). An intrauterine pregnancy was defined on the basis of an ultrasound scan showing an intrauterine gestation sac with or without a visible embryo and heartbeat. Missed miscarriage was confirmed when an empty gestation sac was present with a mean sac diameter of $25 \mathrm{~mm}$ or more, if an embryo with CRL measurement of $7 \mathrm{~mm}$ or more was identified without an embryonic heartbeat or if the embryonic heartbeat was absent irrespective of the size of the CRL, if one had previously been observed [22, 23]. A diagnosis of incomplete miscarriage was made when a transvaginal ultrasound demonstrated irregular heterogeneous tissue in the endometrial cavity in keeping with retained products of conception after a previous ultrasound scan had shown an intrauterine pregnancy [24].

Participants were recruited via open advertisements (using posters) within the hospital and at the university where the study was being conducted (Imperial College). The majority of women were recruited after attending the hospital Ultrasound Department or Early Pregnancy Assessment Unit. Exclusion criteria for this study included women under 18 years of age, sexual intercourse within $72 \mathrm{~h}$ of sampling and human immunodeficiency virus (HIV) or hepatitis C-positive status. All patients using antibiotics, probiotic supplements or progesterone supplements within 2 weeks of sample collection were excluded. A detailed questionnaire including demographic information, past medical, gynaecological and obstetric history was completed. Validated symptom scores were used to assess vaginal bleeding based upon a pictorial blood assessment chart score at the time of sampling [25]. In this methods, bleeding score 0 represents no bleeding, 1 minimal bleeding, 2 moderate bleeding, 3 soaking sanitary towels and 4 passing clots. 


\section{Sample collection}

Cervicovaginal fluid samples were collected from each participant from the posterior vaginal fornix using a BBL CultureSwab MaxV Liquid Amies swab (Becton, Dickinson and Company, Oxford, UK) prior to surgical management of miscarriage (the minimum time between transvaginal ultrasound scan and sample collection was $48 \mathrm{~h}$ ). Swabs were stored in $500 \mu \mathrm{l}$ of liquid amies and immediately placed on ice before being frozen and stored at $-80^{\circ} \mathrm{C}$ within $5 \mathrm{~min}$ of collection. A subset of swabs $(n=96)$ were directly stored at $-80^{\circ} \mathrm{C}$ without liquid amies. All swabs were weighed before and after collection to determine the wet weight. Negative control swabs were also collected by exposing swabs to clinic and laboratory environments prior to freeze storage. The degree of vaginal bleeding was assessed at the time of surgical evacuation.

\section{Cytogenetic analysis}

Chorionic villous material was collected at the time of surgical evacuation of the uterus and analysed for molecular cytogenetics using QF-PCR (quantitative fluorescent polymerase chain reaction) or BACs (bacterial artificial chromosomes) on Beads [26]. For molecular cytogenetics using QF-PCR, DNA was amplified using two multiplexes that include a total of 31 markers; assay 1 contains primers for chromosomes 13, 18, 21 and 22, and assay 2, primers for chromosomes 14, 15 and 16 and the $\mathrm{X}$ and $\mathrm{Y}$ chromosomes. Supplementary markers were used as required. PCR products were separated on an ABI 3100 capillary genetic analyser, and results were analysed using ABI Genotyper software [27]. The KaryoLite bacterial artificial chromosomes-on-Beads (KL$\mathrm{BoBs}^{\mathrm{rm}}$ ) assay was performed using a prenatal chromosome aneuploidy and microdeletion detection test kit (Perkin Elmer, Waltham, MA, USA), according to the manufacturer's instructions. Briefly, genomic DNA from specimens as well as reference DNA were biotinylated and purified. The genomic DNA and $\mathrm{BoBs}^{\mathrm{Tm}}$ was then subjected to single-cell hybridization overnight before washing and incubation with streptavidin-phycoerythrin, which was used as the reporter. Fluorescence of DNA bound to the microbeads was measured using a Luminex 200 (Austin, TX, USA), and BoBsoft $\mathrm{ft}^{\mathrm{tw}}$ analytical software (Perkin Elmer) was used for data analysis whereby a ratio of specimen fluorescence to reference fluorescence greater than 1.0 indicated the chromosome fragments were repeated and a ratio less than 1.0 indicated a deletion [26].

\section{DNA extraction and bacterial 16S rRNA gene amplicon sequencing}

The methods for DNA extraction from the vaginal swabs $\left(\mathrm{BBL}^{\mathrm{TM}}\right.$ CultureSwab ${ }^{\mathrm{TM}}$ ) were followed from those outlined in the Manual of Procedures for the Human Microbiome Project with minor modifications [28]. The details of the DNA extraction from vaginal swabs was performed as previously outlined [12]. In brief, mixed universal primers 28F-YM GAGTTTGATYMTGGCT CAG, 28F-Borrellia GAGTTTGATCCTGGCTTAG, 28F-Chloroflex GAATTTGATCTTGGTTCAG and 28FBifdo GGGTTCGATTCTGGCTCAG at a ratio of 4:1:1: 1 with 388R TGCTGCCTCCCGTAGGAGT reverse primers were used to amplify the V1-V2 region of $16 \mathrm{~S}$ rRNA. Sequencing was performed at RTL genomics (Lubbock, TX, USA) using the Illumina MiSeq platform (Illumina Inc.). The data was processed and analysed using the MiSeq SOP Pipeline of the Mothur package [29]. Sequence alignment was performed using blastn (16SMicrobial.tar.gz) and classification used RDP (Ribosomal Database Project) [30]. To account for sequencing depth bias, data were resampled and normalised to the lowest read count. Rarefaction curves were constructed and analysed to guide selection of depth for rarefaction and Good's coverage index calculated to facilitate assessment of adequacy of sub-sampling of the data.

\section{Cytokine analysis}

The vaginal swabs used for microbiome analysis were thawed slowly on ice and vortexed. For the subset of swabs not stored in amies solution, a total of $500 \mu \mathrm{l}$ of PBS supplemented with $2.5 \mu \mathrm{l}$ protease inhibitor (PI) was added to the swab to provide a comparable dilution volume as those stored in Amies transport solution. A constant volume was used as the mean wet weight for all swabs were highly comparable (mean $0.07 \mathrm{~g} \pm 0.02$ ). The samples were then centrifuged (8000 rpm for $10 \mathrm{~min}$ ) and the supernatant removed and stored at $-20^{\circ} \mathrm{C}$ for cytokine studies. In cases where supernatant was not collected and only dry swabs were available, we suspended the swab in protease inhibitor (PI) and PBS $(5 \mu \mathrm{l}$ $\mathrm{PI} / 1 \mathrm{ml} \mathrm{PBS}$ ) to make up the same volume of as those stored in Amies transport solution. The supernatants $(50 \mu \mathrm{l})$ were analysed by Human Magnetic Luminex Screening Assay (8-plex) to measure the concentration of the following analytes: IL-2, IL-4, IL-6, IL-8, TNF- $\alpha$, IFN- $\gamma$, IL-1 $\beta$, IL-18 and IL-10.

\section{Statistical analysis}

Analysis of statistical differences between the vaginal microbiota of samples according to pregnancy outcome was performed using hierarchical clustering analysis (HCA) using Ward linkage in CLUSTVIS (https://biit.cs. ut.ee/clustvis). Vaginal microbiota composition was classified into two groups at genus level according to the relative abundance of Lactobacillus spp. using Ward hierarachial clustering analysis; samples were also classified into CSTs using the recently developed VAgina $L$ 
community state typ $E$ Nearest CentroId classifier (VALENCIA) [31]. The Fisher's exact test was used when comparing Lactobacillus spp. depleted and dominated in three different pregnancy outcome categories.

Linear discriminant analysis (LDA) effect size (LEfSe) analysis was used to identify taxa significantly overrepresented according to clinical outcome, through all taxonomic levels [32]. This analysis was performed using taxonomic relative abundance, with per-sample normalisation and default settings for alpha values $(0.05)$ for the factorial Kruskal-Wallis test among classes and pairwise Wilcoxon test between subclasses. A logarithmic LDA score $>2$ was used to determine discriminative features.

Other statistical analyses were performed using the statistical package GraphPad Prism v.8.4.3 (GraphPad Software Inc., CA, USA).

To infer the pattern of microbial relationships in the vaginal microbiome, we used BAnOCC [33] to construct correlation matrices at the genus and species level (we limited the latter to the top 50 species with the greatest overall abundance across the entire cohort). The MCMCs were run for 10,000 iterations and four chains (runs were checked for convergence). Co-occurrence networks were drawn using these correlation matrices in the Qgraph package in R [34].

\section{Results}

\section{Patient cohort, characteristics and outcomes}

Two hundred women were recruited, of whom 119 miscarried and 81 had a viable term pregnancy. Of the women who miscarried, 92 were recruited at first consultation, the time of miscarriage diagnosis. A live pregnancy was initially diagnosed in 108 cases of whom 27 went on to miscarry and 81 had a successful pregnancy and delivered at term. Cytogenetic analysis was unavailable in 26 cases (in 24 cases due to inadequate numbers of villi and in two cases because of technical failure). Of the 81 viable pregnancies, eight swabs were unavailable for cytokine analysis and seven unavailable for microbiota analysis. The final study cohort consisted of two patient groups, one miscarriage group for which vaginal microbiota, cytogenetic and vaginal cytokine concentrations were available for all cases and one term pregnancy group for which 74 microbiota data and 73 cytokine data were available. The final cohort therefore comprised a total 93 pregnancies that miscarried and 74 pregnancies that went to term (Fig. 1). Table 1 shows the clinical and demographic characteristics of the three patient groups. Median maternal age was 32 (range 17-46) in the euploid miscarriage group, 36 (range 27-45) in the aneuploid miscarriage group and 32 (range 20-44) in the term pregnancy group. Maternal age was significantly higher in the aneuploid miscarriage group $(P=0.0044$, Kruskal-Wallis test). Women in the term pregnancy group were significantly less likely to have had one or more previous miscarriages $(P=0.0084$, chi-squared test). Gestational age at sampling was categorised as 5$8,8-10$, or $10-14$ weeks post last menstrual period (LMP). There were no significant differences in gestational age at the time of sampling between aneuploid and euploid miscarriage group, but the term pregnancy group were sampled significantly later $(P=0.0083$, chisquared test). Bleeding scores were similar between euploid and aneuploid miscarriage groups and higher bleeding scores were more common in the miscarriage groups compared to the term pregnancy group $(P=$ 0.0002 , chi-squared test). There were no significant differences in BMI, smoking status or ethnicity between the groups.

\section{Baseline vaginal microbiota composition and pregnancy outcomes}

In total 4,523,582 sequence reads were obtained from 167 samples with an average of 27,087 reads per sample and a median read length of $370 \mathrm{bp}$ after bar code removal. Following removal of singletons and rare operational taxonomic units (OTUs), a total of 128 taxa were identified in the vaginal microbiota of the study cohort. All negative control samples failed to amplify or generate any read data following sequencing apart from 1 ,

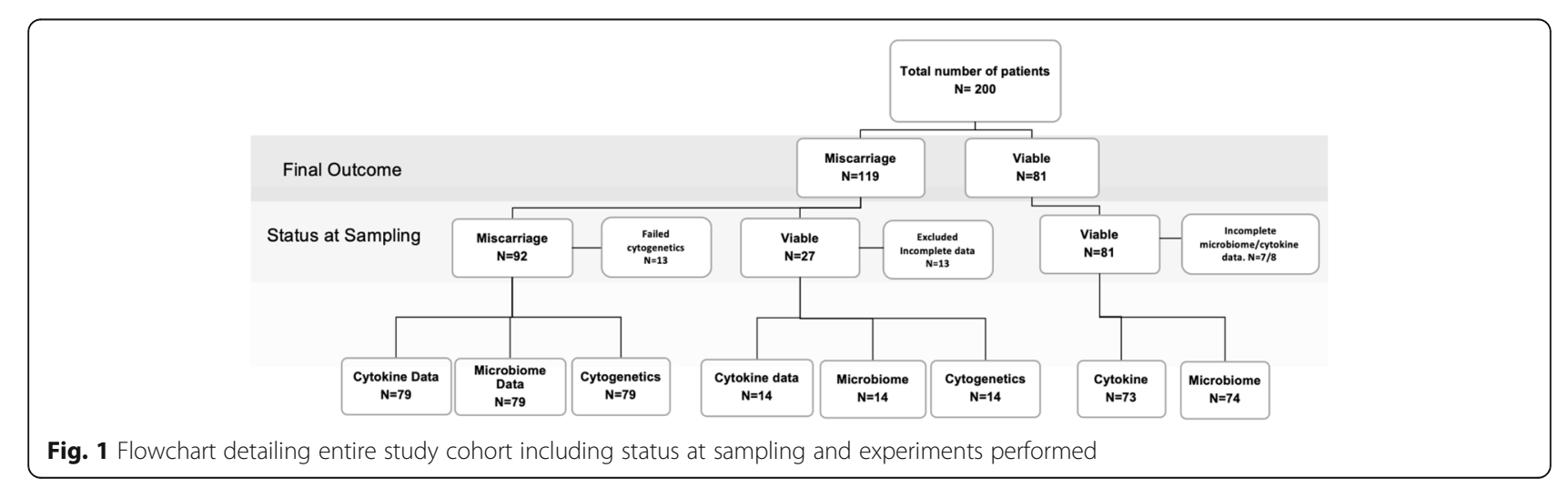


Table 1 Clinical and demographic characteristics of 167 patients included in study cohort

\begin{tabular}{|c|c|c|c|c|}
\hline & Euploid miscarriage & Aneuploid miscarriage & Control (term pregnancy) & $p$ value \\
\hline Number of women & 54 & 39 & 74 & \\
\hline $\begin{array}{l}\text { Maternal age, years } \\
\text { Median (range) }\end{array}$ & $32(17-46)$ & $36(27-45)$ & $32(20-44)$ & ${ }^{\dagger} 0.0044$ \\
\hline $\begin{array}{l}\text { BMI }\left(\mathrm{kg} / \mathrm{m}^{2}\right) \\
\text { Median (range) }\end{array}$ & $25.1(17.7-35)$ & $24.4(18.7-39.4)$ & $24.5(18-38.4)$ & ${ }^{\dagger} 0.205$ \\
\hline Smokers (\%) & $7(13)$ & $2(5.1)$ & $3(3.7)$ & ${ }^{\dagger+} 0.189$ \\
\hline \multicolumn{5}{|l|}{ Ethnicity (\%) } \\
\hline White & $30(56)$ & $24(62)$ & $54(73)$ & \multirow[t]{4}{*}{${ }^{+++} 0.1271$} \\
\hline Asian & $15(28)$ & $11(28)$ & $10(13.5)$ & \\
\hline Black & $7(13)$ & $4(10)$ & $10(13.5)$ & \\
\hline Mixed & $2(3)$ & $0(0)$ & $0(0)$ & \\
\hline \multicolumn{5}{|c|}{ Previous miscarriage (\%) } \\
\hline 0 & $30(55.6)$ & $22(56.4)$ & $31(42)$ & \multirow[t]{4}{*}{${ }^{+t+t} 0.0084$} \\
\hline 1 & $9(16.7)$ & $6(15.3)$ & $29(39)$ & \\
\hline 2 & $9(16.7)$ & $7(18)$ & $10(14)$ & \\
\hline$\geq 3$ & $6(11)$ & $4(10.3)$ & $4(5)$ & \\
\hline \multicolumn{5}{|c|}{ Gestational age group at sampling (\%) } \\
\hline $5-8$ weeks & $14(26)$ & $14(36)$ & $7(10)$ & \multirow[t]{3}{*}{${ }^{++\dagger} 0.0083$} \\
\hline $8-10$ weeks & $24(44)$ & $19(49)$ & $41(55)$ & \\
\hline 10-14 weeks & $16(30)$ & $6(15)$ & $26(35)$ & \\
\hline \multicolumn{5}{|l|}{ Bleeding score } \\
\hline 0 & 28 & 24 & 65 & \multirow[t]{5}{*}{${ }^{+++} 0.0002$} \\
\hline 1 & 13 & 11 & 9 & \\
\hline 2 & 8 & 2 & 0 & \\
\hline 3 & 3 & 2 & 0 & \\
\hline 4 & 2 & 0 & 0 & \\
\hline \multicolumn{5}{|l|}{ Status at sampling } \\
\hline Missed & 40 & 35 & & \multirow[t]{3}{*}{${ }^{++\dagger} 0.1$} \\
\hline Incomplete & 4 & 0 & & \\
\hline Viable & 10 & 4 & & \\
\hline
\end{tabular}

${ }^{\dagger}$ Kruskal-Wallis test

${ }^{+\dagger}$ Fisher's exact (two-tailed)

${ }^{+++}$Chi-squared

${ }^{+++\dagger} \mathrm{Chi}$-squared combining euploid and aneuploid miscarriages and comparing with viable term pregnancies

which had a read count of 15 . By comparison, the average read depth for patient samples was 27,087 (minimum 1402) reads. The sequencing depth was similar across the three groups with an average read count of 25,926 reads for Euploid miscarriage, 25,257 for Aneuploid and 28,899 for Viable ( $p=0.1196$, Kruskall and Wallis test). To avoid sequencing bias, OTUs were randomly sub-sampled to the lowest read count of 1402 which maintained a minimum Good's coverage value of 95.9\% (range 95.9-99.9\%) for all samples. Further analysis was restricted to the top 50 taxa which accounted for $98 \%$ of the total sequence reads in the dataset.

Ward hierarchical clustering of genus level relative abundance data for the whole cohort identified two major vaginal microbiome groups (VMG), which were characterized by Lactobacillus spp. dominated or Lactobacillus spp. depleted (vaginal microbiome grouping 1; Fig. 2). These were observed in $75 \%(125 / 167)$ and $25 \%$ (42/167) of samples respectively. The Lactobacillus spp. dominated group had a mean Lactobacillus content of 94.2\%. The Lactobacillus spp. depleted group had a mean Lactobacillus content of $18.5 \%$. The Lactobacillus spp. deplete cluster was further divided into Gardnerella dominated and non-Gardnerella (vaginal microbiome sub-grouping 1), and these clusters could be further divided into Gardnerella dominant, mixed Lactobacillus/ Gardnerella, Prevotella or Streptococcus dominant (vaginal microbiome sub-grouping 2). Similar analyses were 


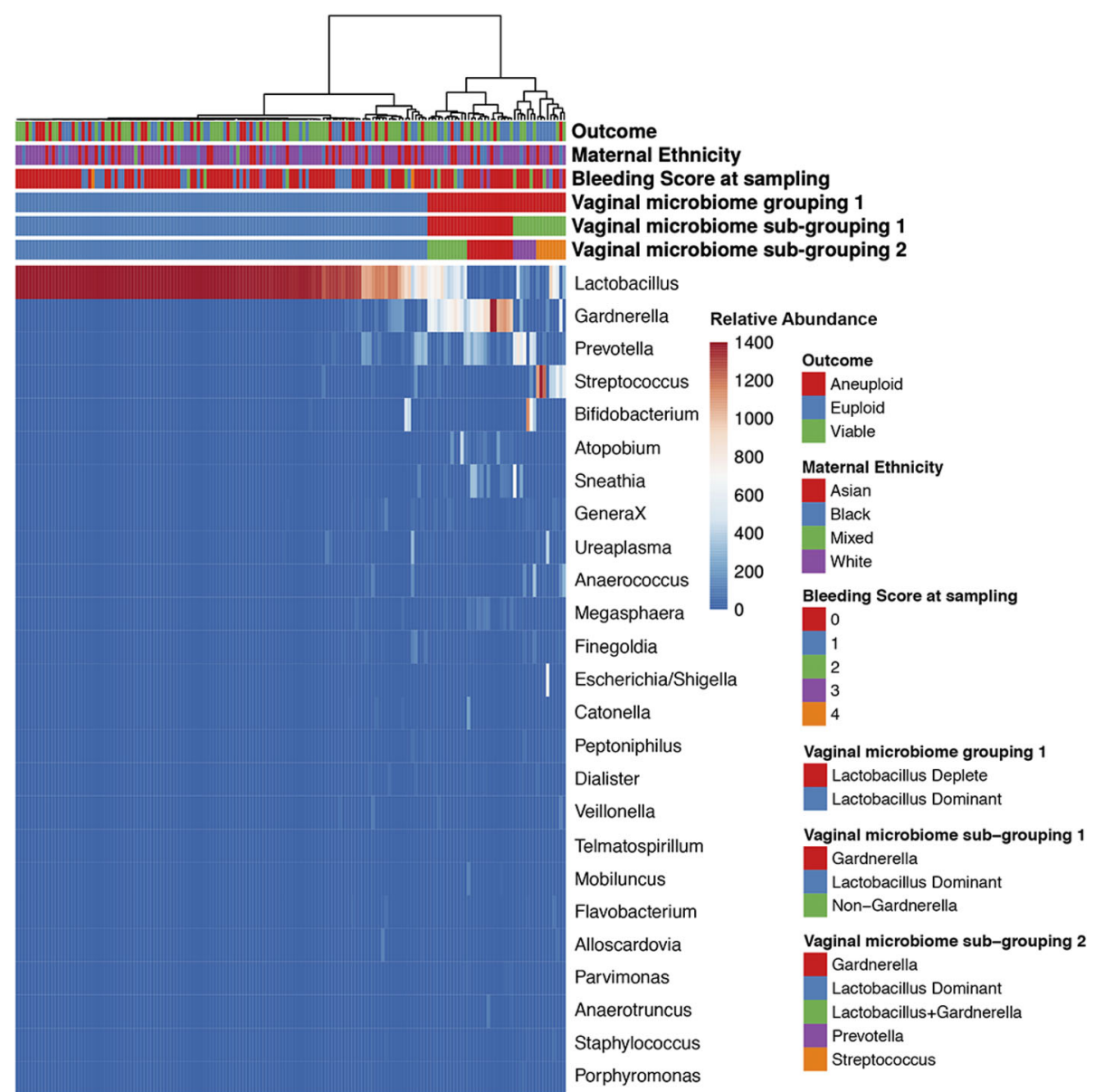

Fig. 2 Heat map of relative abundance data for the top 50 most prevalent vaginal bacterial genera and relationship with different clinical outcomes. Mixed universal primers 28F-YM GAGTTGGATYMGGCTCAG, 28F-Borrellia GAGTTTGATCCTGGCTTAG, 28F-Chloroflex GAATTGATCTTGG TTCAG and 28F-Bifdo GGGTTCGATTCTGGCTCAG at a ratio of 4:1:1:1 with 388R reverse primers were used to amplify the V1-V2 region of 16S rRNA followed by sequencing using the Illumina MiSeq platform. The data was processed using the MiSeq SOP Pipeline of the Mothur package and classification used RDP. Ward hierarchical clustering of genus level relative abundance data for the whole cohort of 167 individual samples identified two major vaginal microbiome groups (VMG), characterized by Lactobacillus spp. dominance 75\% (125/167) and Lactobacillus spp. depletion 25\% (42/167). The mean Lactobacillus content in the Lactobacillus spp. dominated group was $94.2 \%$ and in the Lactobacillus spp. deplete group was 18.5\%. The Lactobacillus spp. deplete cluster was further divided into Gardnerella dominant and non-Gardnerella (VMG subgrouping 1), and these clusters could be further divided into Gardnerella dominant, mixed Lactobacillus/Gardnerella, Prevotella or Streptococcus dominated (VMG sub-grouping 2)

performed at species level to identify the principal Lactobacillus spp. present in the Lactobacillus spp. dominant group of each individual patient. Ward hierarchical clustering separated patient samples into clusters that were dominated by either Lactobacillus crispatus (37\%), Lactobacillus iners (19\%), Lactobacillus gasseri (11\%), Lactobacillus jensenii (10\%), Lactobacillus acidophilus (2\%), Gardnerella vaginalis (15\%) and a highly diverse group (8\%), Additional file 1: Figure S1. Using this classification, no particular Lactobacillus spp. was especially protective (chi-squared, $P=0.3$ ). Classification of samples into equivalent CSTs using the recently developed VALENCIA classification tool [31] indicated broad agreement between the two clustering approaches (84\%)
(Additional file 1: Figure S1). In the remaining samples where classification differed, low similarity scores indicated poor fitting to the CSTs pre-defined by the VALENCIA algorithm. This indicated underlying composition differences in samples from our patient cohort and those used to train VALENCIA. The remaining analyses were therefore performed using our defined community clusters.

Analysis of co-occurrence between bacterial genera within our dataset identified a strong relationship between bacterial vaginosis (BV) associated genera including Gardnerella, Atopobium and Prevotella (Fig. 3a). In contrast, Lactobacillus was negatively correlated with most other genera, especially Gardnerella, Sneathia, 

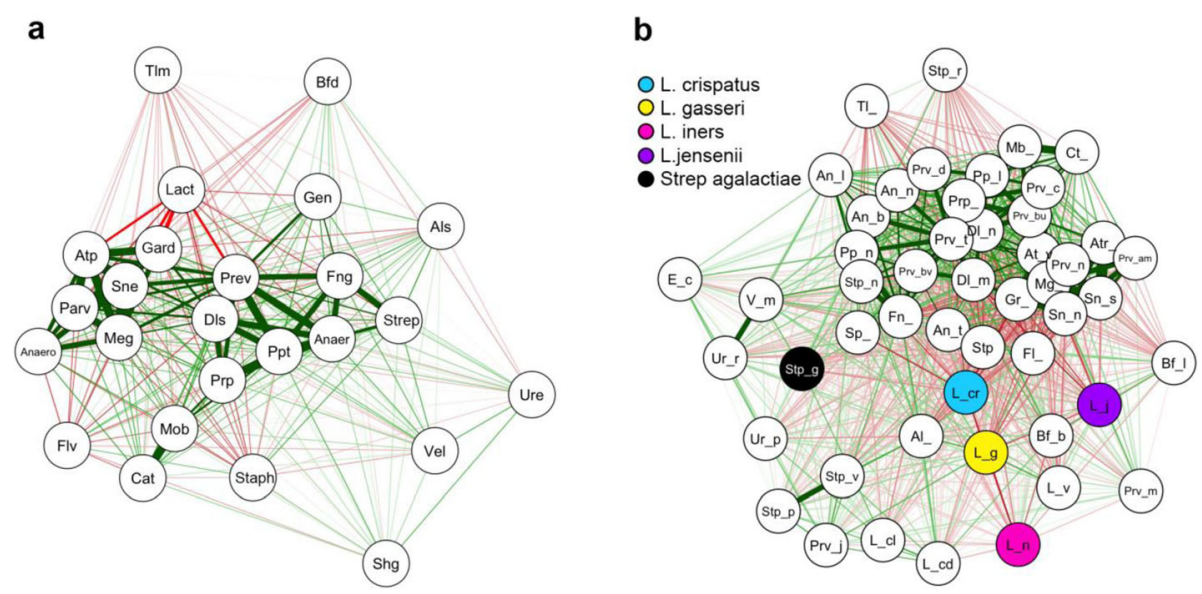

Fig. 3 Co-occurrence network analysis of vaginal bacterial taxa in the overall patient cohort. Bayesian Analysis of Compositional Covariance (BAnOCC) was used to generate co-occurrence networks between vaginal microbiota at genera (a) and species level taxonomy (b) using the 50 species with the highest overall abundance. This programme uses a Bayesian framework to analyse compositional covariance. We ran the MCMC for 10000 generations and four chains and restricted subsequent analyses to edges with $[r]>0.3$. The red lines indicate negative correlations and the green lines indicate positive correlations and the lines are weighted according to the magnitude of the correlation. There were high levels of co-occurrence between BV associated genera, whereas Lactobacillus was negatively correlated with most other genera (a). At species level, L.crispatus had the most number of negative edges with all other bacterial taxa (b)

Atopobium and Megasphaera. When analysis was undertaken at the species level BV-associated taxa were again observed to positively correlate whereas Lactobacillus species, especially $L$. crispatus, tended to be negatively correlated with other bacterial taxa, including with other Lactobacilli. Strong co-occurrence between Streptococcus vestibularis and Streptococcus pneumoniae was also observed and Streptococcus urinalis showed a high density of negative edges with BV-associated bacteria but a positive edge with Streptococcus agalactiae (Fig. 3b).

We next compared vaginal microbiota composition between women who miscarried according to the pregnancy karyotype. Euploid miscarriage was associated with a significantly higher proportion of Lactobacillus spp. deplete VMG when compared to aneuploid miscarriage ( $P=0.01$, two-tailed Fisher's exact test; Fig. 4a). This difference remained significant when correcting for bleeding score by removing patients with a bleeding score $>1(P=0.02$, two-tailed Fisher's exact test; data not shown). Euploid miscarriage was characterised by a significantly higher proportion of non-Gardnerella Lactobacillus spp. depleted VMG $(P=0.02$, two-tailed Fisher's exact test, Figure $4 \mathrm{~b}$ ) which was enriched for Streptococcus spp. in 60\% of cases and Prevotella spp. in $40 \%$ of cases (Fig. 4c). Consistent with these findings, both bacterial richness and alpha diversity were higher in the euploid miscarriage group (Fig. 4d, e). LEfSe analysis similarly identified decreased levels of Lactobacillus and increased levels of Prevotella, Bacteriodia, Clostridia and Dialister as characteristic features of euploid miscarriage compared to aneuploid miscarriage (Fig. 4f, g).
In our cohort, 26 women had at least two previous miscarriages, prior to a further miscarriage in the index pregnancy and were therefore defined as having 'recurrent miscarriage' [35]. Within these cases, there was no difference in the proportion of Lactobacillus spp. deplete VMG or Lactobacillus spp. dominant VMG between euploid and aneuploid miscarriage (Fig. 5b). Within the sporadic miscarriage and viable term pregnancy group the proportion of Lactobacillus spp. deplete VMG was greater in the euploid miscarriage group than either aneuploid miscarriage or viable pregnancy groups $(P=$ 0.02 and $P=0.05$ respectively, two-tailed Fisher's exact test, Fig. 5a). The prevalence of non-Gardnerella Lactobacillus spp. depleted VMG was also greater in the euploid miscarriage group than either aneuploid miscarriage or viable pregnancy groups $(P=0.03$ and $P$ $=0.04$ respectively, two-tailed Fisher's exact test, Fig. $5 \mathrm{c}$ ) and was particularly associated with Streptococcus spp. dominated compositions (Fig. 5d). Although the prevalence of Lactobacillus spp. depleted VMG was greater in the euploid miscarriage than the viable pregnancy groups, richness and diversity were not significantly different between the two groups. Similar results were obtained when re-analysis was performed on only those women where recurrent miscarriage was defined as being three consecutive miscarriages with no live births ( $n$ = 13, Additional file 1: Figure S2) [36]. LEfSe analysis identified increased relative abundance of Prevotella and Streptococcus spp. and reduced relative abundance of Lactobacillus spp. as being discriminatory for sporadic euploid miscarriage compared to viable term pregnancies (Fig. 5e). 


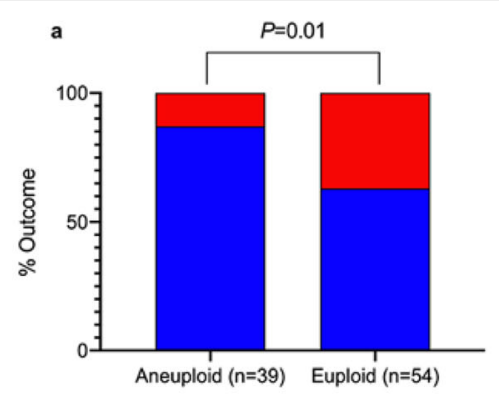

Lactobacillus spp. $\square$ dominant $\square$ deplete
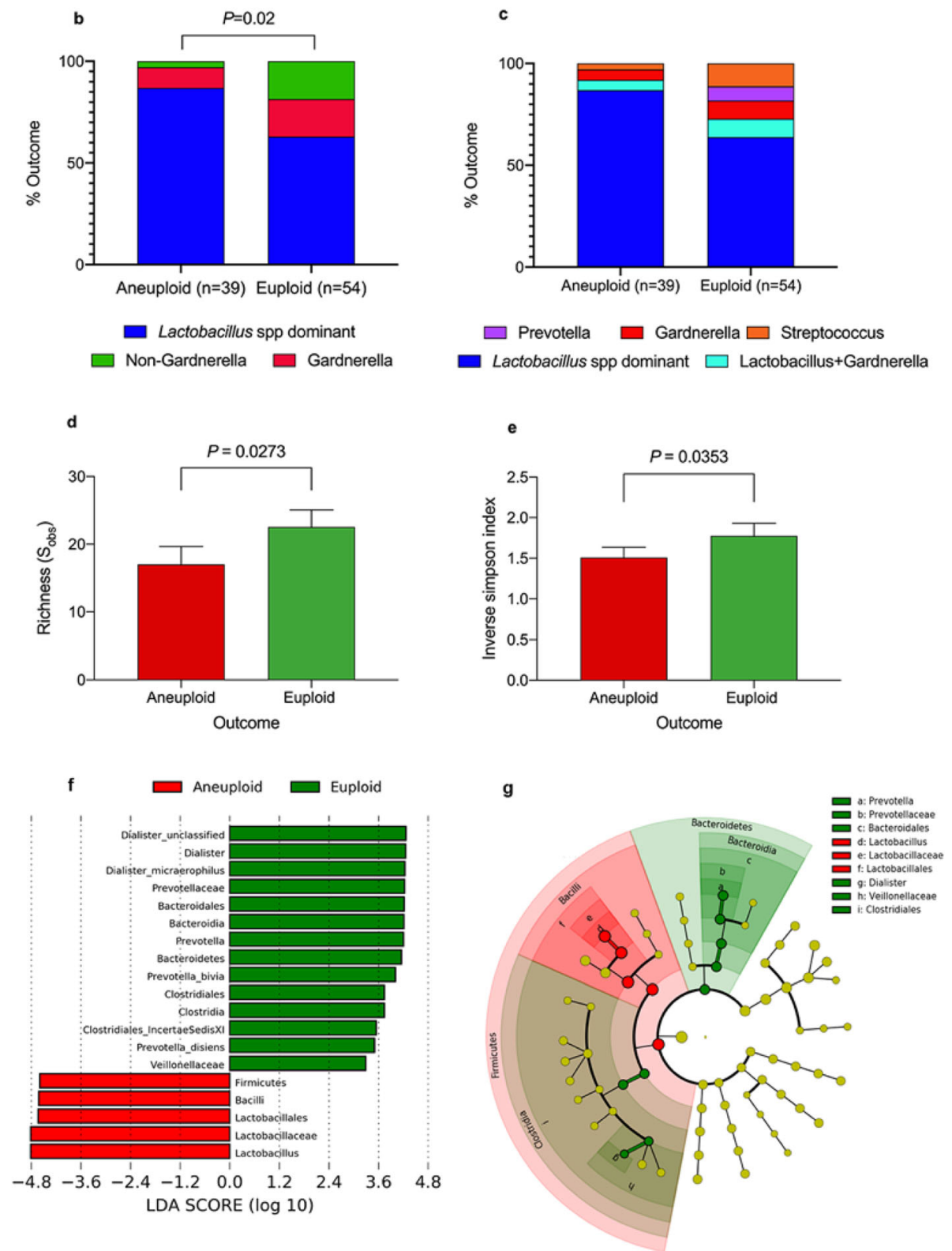

Fig. 4 (See legend on next page.) 
(See figure on previous page.)

Fig. 4 Miscarriage outcome according to vaginal microbial classification. Stacked bar chart illustrating increased representation of Lactobacillus spp. depleted vaginal microbial communities in euploid miscarriages compared to aneuploid miscarriages $(P=0.01$, two tailed Fisher's exact test a). Analysis of vaginal microbiome subgroups showed that this difference was largely driven by non-Gardnerella-dominance of the vaginal niche $(P=0.02$, two-tailed Fisher's exact test $\mathbf{b})$ and increased relative abundance of Streptococcus and Prevotella species in euploid miscarriages compared to aneuploid miscarriages $(\mathbf{c})$. Consistent with this, significantly higher richness $(P=0.0273$, two-tailed Mann-Whitney $U$, d) and diversity $(P=0.0353$, two-tailed Mann-Whitney $U$, e) was observed in euploid miscarriage compared to aneuploid miscarriage. The linear discriminant analysis (LDA) effect size (LEFse) method (f) was then used to identify differentially abundant taxa in euploid compared to aneuploid miscarriage, which were presented at differing taxonomic levels using a cladogram $(\mathbf{g})$. Data represented as percentages $\mathrm{a}, \mathrm{b}$ and $\mathrm{c}$ and mean \pm standard error of mean in $\mathbf{d}$ and $\mathbf{e}$. LEfSe analysis $(\mathbf{f}, \mathbf{g})$ depicting particular vaginal microbial taxa associated with different clinical outcomes

\section{Relationship between vaginal microbiota and cytokines concentrations across the entire cohort}

To explore the relationship between the vaginal microbiota and cervicovaginal inflammatory markers, we compared the levels of nine cytokines between women with Lactobacillus spp. depleted or Lactobacillus spp. dominated VMG irrespective of pregnancy outcome. The Lactobacillus spp. depleted non-Gardnerella VMG subgroup had significantly higher concentrations of tumour necrosis factor (TNF)- $\alpha$, interleukin (IL)-6, IL-1 $\beta$ and IL-18 compared to Lactobacillus spp. dominated VMG $(P=0.001,0.045,0.04,0.04$ respectively, Fig. 6a). The individual data points for these four cytokines (TNF- $\alpha$, IL-6, IL-1 $\beta$ and IL-18), their positions within the concentration range quartiles and their relationship to the proportion of Lactobacillus spp. relative abundance in the different pregnancy outcome groups is shown in Fig. $6 \mathrm{~b}-\mathrm{e}$. High cytokine levels for TNF- $\alpha$ and IL-1 $\beta$ (defined as concentrations in the upper quartile) were more frequently observed in the Lactobacillus spp. depleted group $(P=0.009$ and 0.002 respectively, two-tailed Fisher's exact test, Fig. 6f), particularly non-Gardnerella dominated samples, which also had elevated IL6 levels ( $P=0.009$, two-tailed Fisher's exact test, Fig. 6g). Further subdivision of the VMG groups showed that increased levels of TNF- $\alpha$ and IL-1 $\beta$ were largely associated with Prevotella spp. dominance $(P=0.007$ and 0.018 respectively, chi-squared test) whereas IL6 was linked to Streptococcus dominance $(P=0.02$ chi-squared test, Fig. $6 \mathrm{~h})$.

\section{Relationship between vaginal microbiota, cytokine concentrations and pregnancy outcome}

The proportion of Lactobacillus spp. depleted vaginal microbiota in the aneuploid miscarriage, euploid miscarriage and viable pregnancy groups was, $13 \%, 37 \%$ and $23 \%$ respectively. Since nearly a quarter of the pregnancies with a viable outcome nevertheless had a Lactobacillus spp. depleted vaginal microbiota, we next compared cytokine concentrations between euploid miscarriage and viable term pregnancies in only women with Lactobacillus spp. depletion. IL-1及, IL-6 and IL-8 were significantly lower $(P<0.001, P<0.001$ and $P=$ 0.01 , respectively, two tailed Mann-Whitney $U$ test) and
IL-2 and IL-10 were significantly higher $(P=0.004$ and $P<0.001$ respectively, two tailed Mann-Whitney $U$ test) in viable pregnancies (Fig. 7).

\section{Discussion}

We confirmed our primary hypothesis that euploid miscarriage is significantly more frequently associated with Lactobacillus spp. depleted vaginal microbial communities compared to aneuploid miscarriage. This association was reflected in increased richness and diversity in euploid miscarriage. A Lactobacillus spp. depleted vaginal microbiota characterized by Streptococci was the most significant vaginal microbiota risk factor for sporadic euploid miscarriage and the principal driver of proinflammatory mediators in these patients. We also found that aneuploid miscarriages and healthy pregnancies had similar vaginal microbial compositions. These data support the notion that aneuploid and euploid miscarriages generally have different causal mechanisms. In general, aneuploid miscarriage is assumed to be due to a genetic intrinsic failure whereas a proportion of sporadic euploid miscarriage are due to host-vaginal microbe interactions.

Of the 200 patients recruited to this study, 119 eventually miscarried and 81 went on to have a viable pregnancy. The relatively high rate of miscarriage in this cohort was because the majority of miscarriage patients $(n=92)$ were recruited at the time of miscarriage diagnosis. Molecular cytogenetics was available for 93 miscarriage patients, and results were reported after recruitment and sample collections, therefore eliminating selection bias. The proportion of aneuploid pregnancies in our miscarriage cohort is comparable with the aneuploidy rate reported in previous studies [37, 38]. In our cohort, there was a significantly higher maternal age in the aneuploidy miscarriage group, which is consistent with the known relationship between maternal age and the incidence of meiotic errors in embryos [39]. There was a significant difference in the gestational age at sampling between the viable pregnancies and miscarriages likely because women experiencing symptoms in a viable pregnancy tend to present at a later gestational age to the clinic. As expected, there was a significantly higher bleeding score in the miscarriage cohort compared to the viable term group [21]. Recurrent miscarriage is 


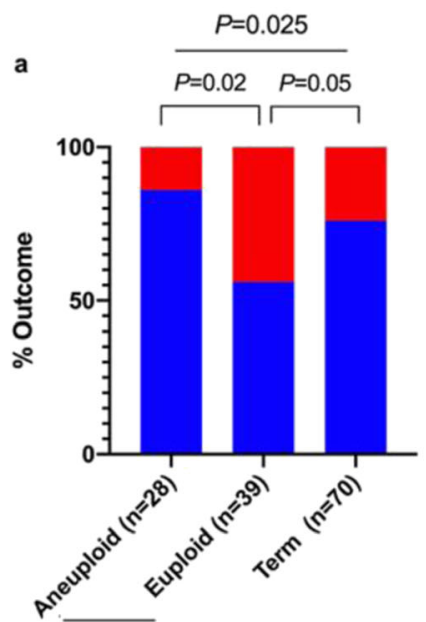

Sporadic miscarriage
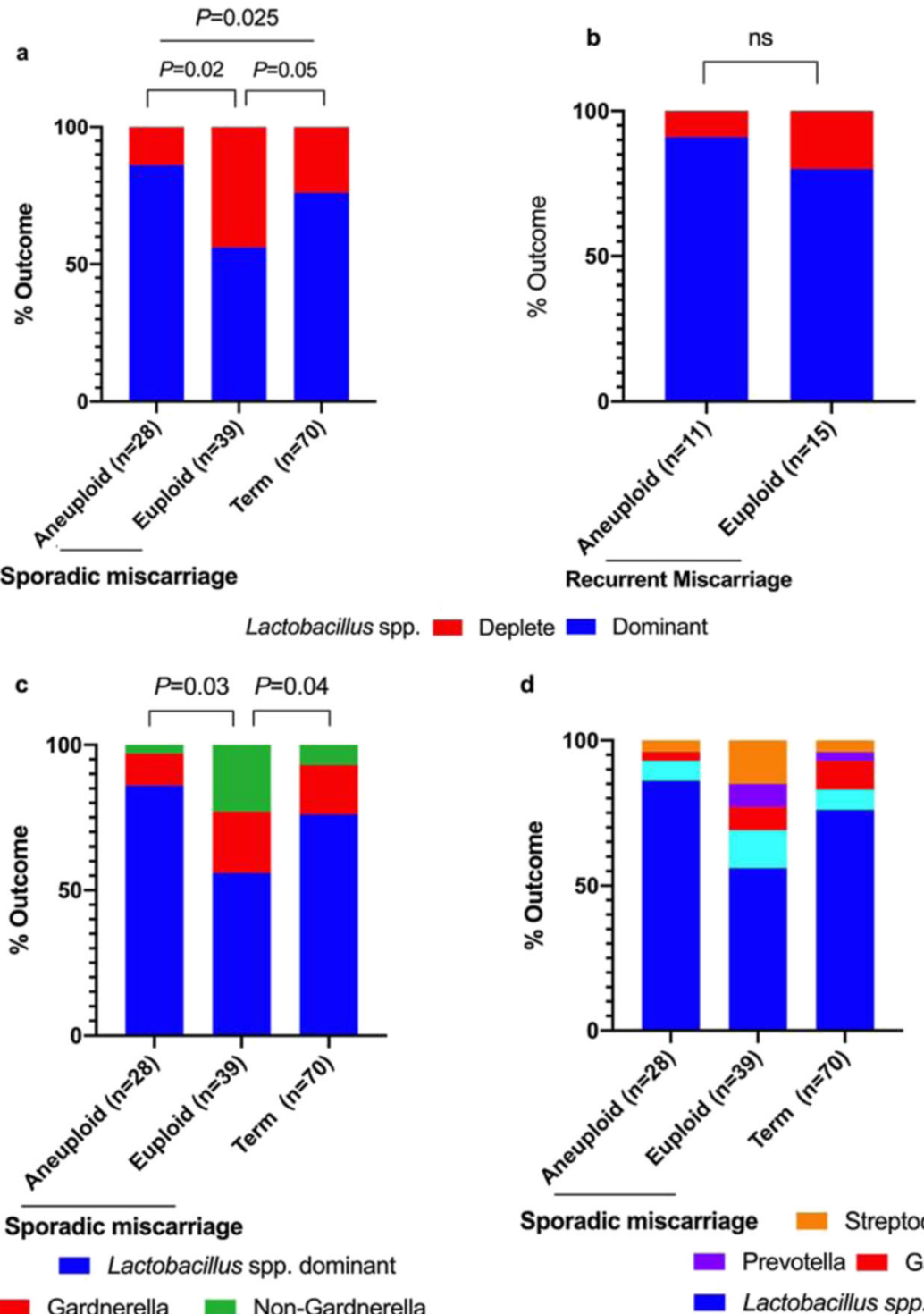

d
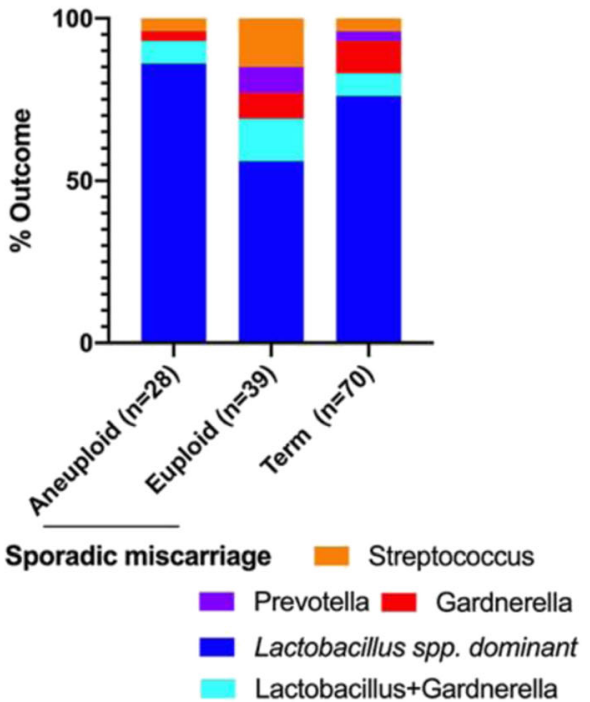

e

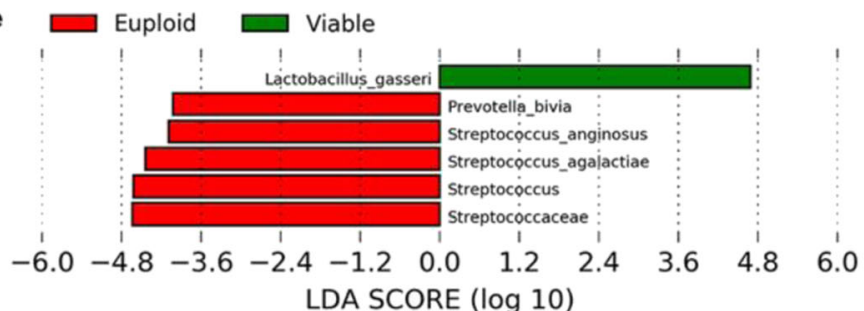

Fig. 5 Clinical outcomes according to vaginal microbial composition in sporadic miscarriage. Increased Lactobacillus spp. depleted vaginal microbial communities were observed in sporadic euploid miscarriages $(n=39)$ compared to sporadic aneuploid miscarriages $(n=28, P=0.02$ two-tailed Fisher's exact test) and viable term pregnancies $(n=70 P=0.05$ two-tailed Fisher's exact test, a). No significant difference was seen between euploid $(n=15)$ and aneuploid $(n=11)$ miscarriages in the recurrent miscarriage group $(\mathbf{b})$. A significantly increased prevalence of nonGardnerella vaginal bacterial communities was seen in euploid miscarriages compared to aneuploid miscarriage, $P=0.03$ and viable pregnancy $P$ $=0.04$ (two-tailed Fisher's exact test, $\mathbf{c}$ ). Differentially abundant taxa identified by LDA in euploid miscarriage compared to viable pregnancy are shown in e. Data represented as percentages in $\mathbf{a}, \mathbf{b}, \mathbf{c}$ and $\mathbf{d}$. LEfSe analysis (e) depicting particular vaginal bacterial taxa associated with euploid and viable pregnancy, confined to sporadic miscarriage 

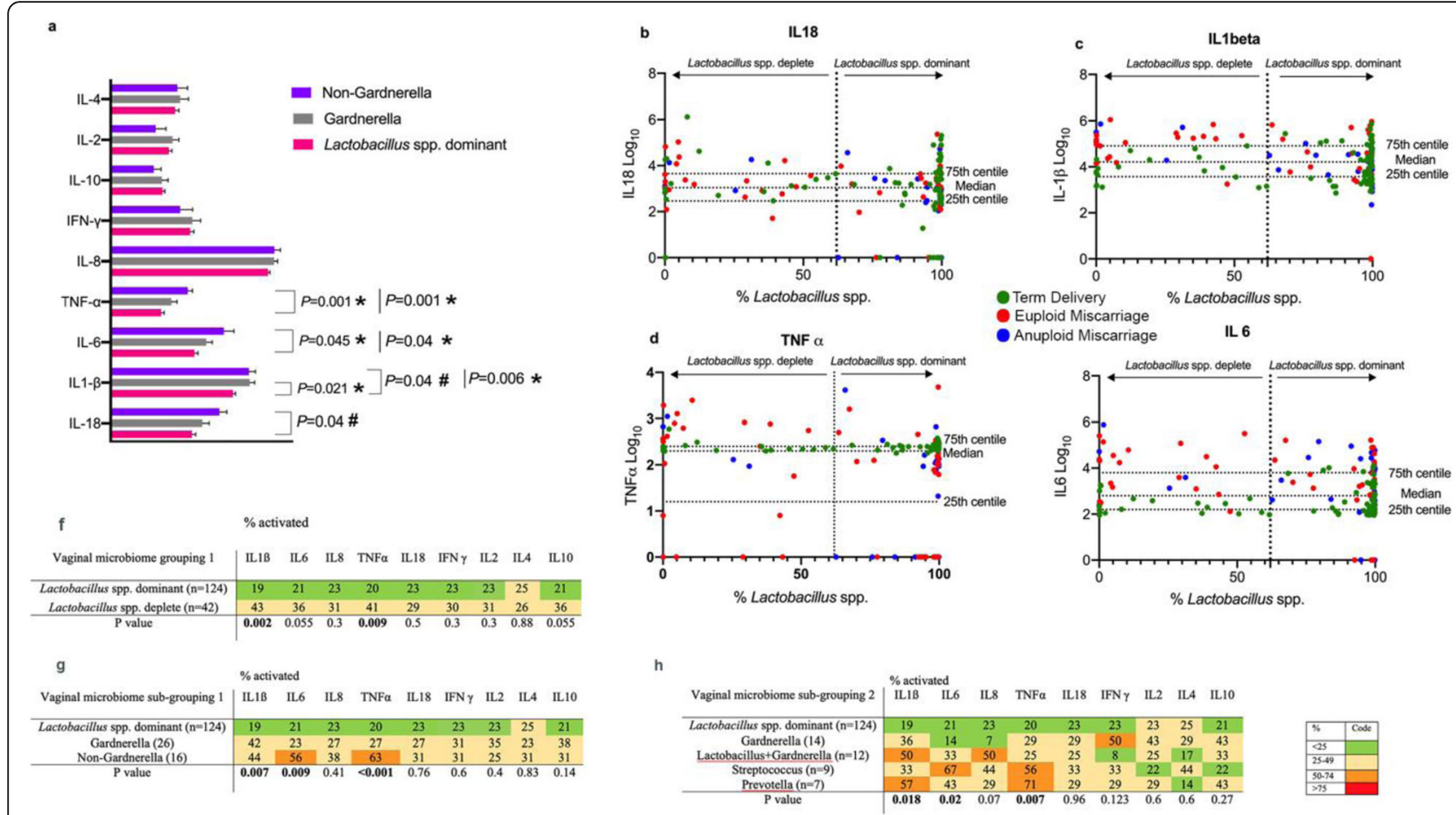

Fig. 6 Cytokine expression according to vaginal microbiome grouping 1 and subgroupings 1 and 2. Non-Gardnerella, Lactobacillus spp. depleted samples (16/166) had significantly increased vaginal levels of inflammatory cytokines TNF-a, IL-6, IL-1 $\beta$ and IL-18 when compared to Lactobacillus spp. dominant samples (124/166). Gardnerella, Lactobacillus spp. depleted samples (26/166) had significantly increased IL-1 $\beta$ when compared to Lactobacillus spp. dominant (a, ${ }^{*} P$ values, Kruskal-Wallis test with post hoc Bonferroni correction and ${ }^{*} P$ values, Mann-Whitney $U$ test for nonGardnerella versus Lactobacillus spp. dominant patients). Scatter plots representing the percentage of Lactobacillus spp. against the concentration of IL-18 (b), IL-1 $\beta$ (c), TNF-a (d), IL-6 (e) and coloured according to different clinical outcomes with the 25th, median, and 75th centile of cytokine levels indicated. Lactobacillus spp. depleted samples had significantly increased levels of IL-1 $\beta$ and TNF-a compared to the Lactobacillus spp. dominated group (f). Increased inflammatory activation in the Lactobacillus spp. depleted samples could be largely attributed to non-Gardnerella dominated samples $(\mathbf{g})$ and more specifically, high relative abundances of Streptococcus and Prevotella spp. (h). Data represented as a clustered bar chart with mean \pm standard error of the mean for each cytokine by vaginal microbiome sub-grouping $1, n=166$ (a). The individual tables (fh) illustrate the percentage of activated cytokines in each group. Activation was defined by cytokines expressed in the upper quartile. $P$ values obtained by comparing activated versus non-activated cytokines using two-tailed Fisher's exact test (f and $\mathbf{g}$ ) and chi-squared test (h)

thought to be aetiologically different to sporadic miscarriage and recent studies have highlighted the association with abnormal endometrial function, caused by lack of mesenchymal stem cells and heightened cellular senescence during the midluteal implantation window [4042]. Our data supports the relationship between miscarriage and vaginal microbiota composition principally in sporadic miscarriage. Although the numbers of recurrent miscarriages in our cohort were small $(n=26)$ and the findings require confirmation in larger populations, this does reinforce the concept that different underlying causal mechanisms drive recurrent miscarriage.

The vaginal microbiota is an important regulator of innate immune response in the reproductive tract [18].

A significant body of evidence has linked inflammation within the cervicovaginal niche to adverse pregnancy outcomes, particularly in relation to second trimester pregnancy loss and preterm birth [43-47]. Consistent with previous findings in pregnant [18] and nonpregnant women [48, 49], we found that proinflammatory cytokines IL-1 $\beta$, IL- 6 and TNF- $\alpha$ were elevated in women with Lactobacillus spp. depleted VMGs. These findings support our primary hypothesis and reinforce the view that Lactobacillus spp. depleted vaginal microbiota correlate with local inflammatory activation which during pregnancy, can associate with adverse pregnancy outcome [18]. However, a Lactobacillus spp. depleted VMG was compatible with delivery at term in nearly a quarter of cases. Our exploration of local immune mediators revealed that women with a Lactobacillus spp. depleted VMG who delivered at term had comparably lower levels of proinflammatory cytokines in early pregnancy. Further, although no difference in the levels of anti-inflammatory cytokines IL-2 and IL-10 were observed between Lactobacillus spp. depleted and Lactobacillus spp. dominated VMGs across the whole cohort, there were significantly increased levels of these cytokines in Lactobacillus spp. depleted samples from viable term pregnancies compared to euploid miscarriage. Collectively, these observations implicate both adverse 


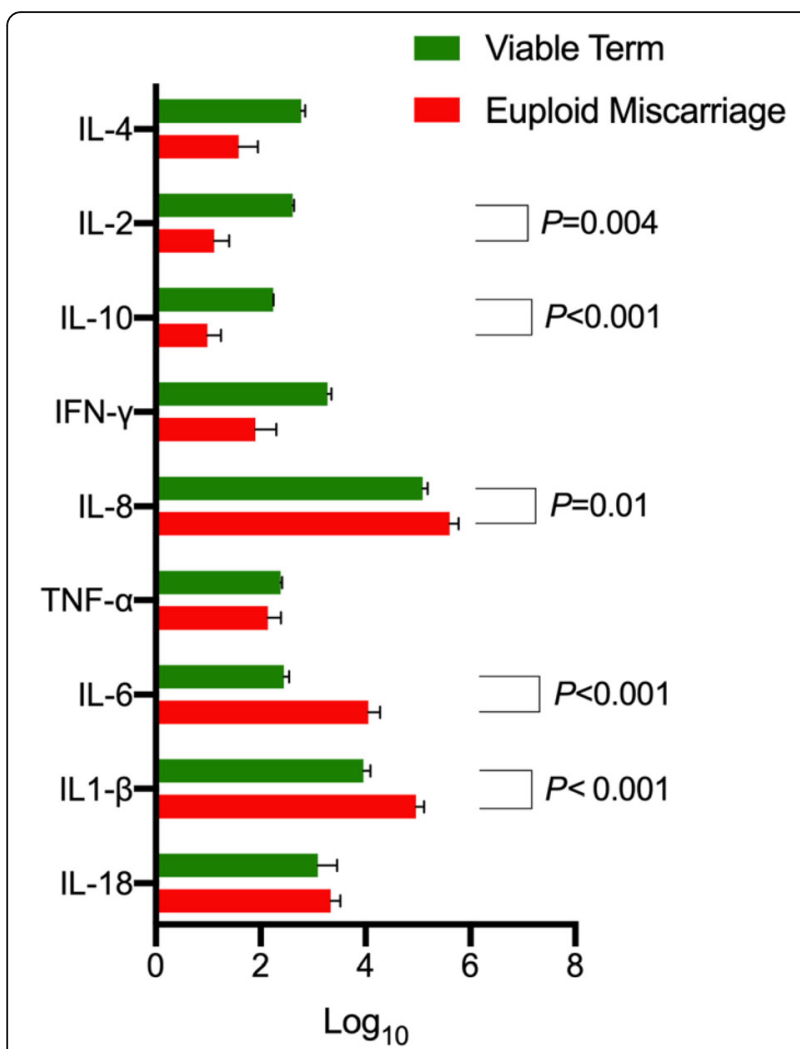

Fig. 7 Vaginal cytokine levels according to pregnancy outcome in patients with Lactobacillus spp. depleted vaginal microbial composition ( $n=37$, euploid $n=20$ and viable term $n=17$ ). In women with Lactobacillus spp. depleted vaginal microbial compositions, viable pregnancy was associated with significantly higher levels of anti-inflammatory cytokines (IL-2 $P=0.004$, IL-10 $P<$ 0.001 , two tailed Mann-Whitney $U$ test) and lower levels of proinflammatory cytokines (IL-6, $P<0.001, \mathrm{IL}-1 \beta P<0.001$, and IL-8 $P=$ 0.01, two tailed Mann Whitney $U$ test) compared to euploid miscarriage. Data represented as clustered bar chart with mean \pm standard error of the mean for each cytokine according to clinical outcome

vaginal microbiota composition and specific local host immune responses in early pregnancy with subsequent risk of miscarriage.

In this study, Gardnerella vaginalis was observed as being an important feature in high risk Lactobacillus spp. deplete compositions. Recent work has indicated the likely existence of difference Gardnerella vaginalis clades with potentially varying degrees of pathogenicity [50]. However, the metaxonomics approach used in our study was not capable of differentiating these. Future investigations may shed light on whether particular Gardnerella vaginalis clades are responsible for the observed relationship with adverse outcomes.

Furthermore, in our cohorts increased production of proinflammatory cytokines (IL-6, IL-1 $\beta$, IL-18 and TNF$\alpha)$ within the Lactobacillus spp. depleted VMGs was associated with dominance by Prevotella and Streptococcus species in women with euploid miscarriages. These species had low co-occurrence with L. crispatus, which has been shown to associate with vaginal bacterial stability and low levels of innate immune activation during pregnancy $[18,51]$. Thus, whilst the underlying microbial structure may be the primary driver, it is the interplay between the vaginal microbiota and host immune response that determines the amplitude of the inflammatory response and the likelihood of miscarriage.

There are a number of potential mechanisms by which vaginal microbiota and host immune response could be causally linked to miscarriage. Suboptimal vaginal microbiota characterised by Lactobacillus spp. depletion and high bacterial diversity are associated with local inflammatory activation and damage to the cervical epithelial barrier [52] that can promote bacterial translocation [53]. Although studies of the endometrial microbiome are confounded by the difficulties of contamination and low biomass, the emerging evidence is that the lower uterine microbiome is distinct but maybe contributed to by the vaginal microbiota $[54,55]$. In this study, microbiota and cytokine measurements were limited to CVF, thus it is not possible to determine if our observations are reflective of microbiota-host interactions in the endometrial mucosa. Logistical and ethical considerations make direct sampling of the early pregnancy uterine environment difficult. However, the embryological origin of Mullerian duct fusion means that the upper one third of the vagina shares similarities with the endometrial epithelium and therefore a similar proinflammatory response to pathobionts such as Prevotella and Streptococcus species would be expected $[56,57]$.

Lactobacilli confer protection in the vagina by promoting antimicrobial defense without initiating innate immune mediated inflammation [14]. Therefore it is possible in cases of Lactobacillus spp. depletion a proinflammatory environment can alter successful implantation, which is a process of tissue injury and repair that is regulated by immune mediators to allow the trophoblast to breach the decidual lining and invade the maternal decidua [5, 58]. Previous studies have also shown that altered levels of cytokines at the maternal fetal interface can trigger early pregnancy complications [5961].

It is therefore plausible that an inflammatory response triggered by the vaginal microbiome could directly or indirectly contribute to dysregulation of the maternal decidua, promoting breakdown of the nascent maternalfetal interface in early gestation [9].

\section{Conclusions}

In conclusion, we demonstrate that vaginal microbiota depleted of Lactobacillus spp. combined with a heightened local inflammatory response, predispose pregnant 
women to euploid miscarriage. Although this may be a reflection of intrinsic maternal immune response, it appears that the cytokine response is largely driven by the bacterial taxa present in the vagina, which presents an opportunity for specific, directed intervention prior to conception or in early pregnancy. First trimester miscarriage occurs in $20 \%$ of pregnancies and is a major cause of physical and psychological pathology worldwide [62]. Whilst aneuploid miscarriage can be explained in terms of an intrinsic developmental defect, there is currently little evidence to explain the causative mechanisms underlying sporadic euploid miscarriage. There are currently no treatments to prevent this important clinical condition. The data presented here suggests that there is a group of women who would benefit from antibiotic or pre- or probiotic treatment to reduce the risk of miscarriage. Whilst further studies are needed to validate these findings and to understand what specifically triggers an inflammatory cascade in euploid miscarriage, an important next step will be to explore which interventional regimes might change the vaginal microbiota and positively influence pregnancy outcome.

\section{Abbreviations}

PPROM: Preterm prelabour rupture of fetal membranes; CVF: Cervicovaginal fluid; LMP: Last menstrual period; CRL: Crown-rump length; QF-

PCR: Quantitative fluorescent polymerase chain reaction; BACs: Bacterial artificial chromosomes; OUT: Operational taxonomic units; VMG: Vaginal microbiome groups; BMI: Body mass index; DNA: Deoxyribonucleic acid; LDA: Latent discriminatory analysis; LEfSe: Linear discriminant analysis with effect size

\section{A.Additional files}

\section{Acknowledgements}

We would like to acknowledge all the participants involved in the study and Tommy's miscarriage charity for funding the research.

\section{Authors' contributions}

PRB, DAM and TB contributed to the conception and design of the study. Ethical approval, recruitment and sample collection was undertaken by KG and MA-M. Experiments and data collection were performed by KG and YSL. Data analyses and interpretation of results was performed by KG, AS, PRB, SK, $J J B$ and DAM. The Figures and tables were generated by KG, SK and PRB. The manuscript was written by KG, DAM and PRB. All authors read and approved the final manuscript.

\section{Funding}

This work was supported by Tommy's National centre for miscarriage research (grant P62774)

\section{Availability of data and materials}

The datasets used and/or analysed during the current study are available from the corresponding author on reasonable request.

\section{Declarations}

\section{Ethics approval and consent to participate}

The study was approved by NHS National Research Ethics Service (NRES) (REC 16/WA/0357 and REC 14/LO/0199). All participants provided written informed consent.

\section{Consent for publication}

Not applicable.

\section{Competing interests}

The authors have no financial, commercial, personal, or professional competing interests to declare.

\section{Author details}

${ }^{1}$ Tommy's National Centre for Miscarriage Research, Institute of Reproductive \& Developmental Biology, Imperial College London, Hammersmith Hospital Campus, Du Cane Road, London, UK. ${ }^{2}$ March of Dimes, European Preterm Birth Research Centre, Imperial College London, Hammersmith Hospital Campus, Du Cane Road, London, UK. ${ }^{3}$ Faculty of Health and Applied Sciences, University West of England, Bristol, UK. ${ }^{4}$ Tommy's National Miscarriage Research Centre, Division of Biomedical Sciences Warwick Medical School, University of Warwick, Coventry, UK.

Received: 12 May 2021 Accepted: 29 December 2021

Published online: 28 January 2022

\section{References}

1. Eiben B, Bartels I, Bahr-Porsch S, Borgmann S, Gatz G, Gellert G, et al. Cytogenetic analysis of 750 spontaneous abortions with the directpreparation method of chorionic villi and its implications for studying genetic causes of pregnancy wastage. Am J Hum Genet. 1990;47(4):656-63.

2. Giakoumelou S, Wheelhouse N, Cuschieri K, Entrican G, Howie SE, Horne AW. The role of infection in miscarriage. Hum Reprod Update. 2016;22(1): 116-33. https://doi.org/10.1093/humupd/dmv041.

3. Coomarasamy A, Harb HM, Devall AJ, Cheed V, Roberts TE, Goranitis I, et al. Progesterone to prevent miscarriage in women with early pregnancy bleeding: the PRISM RCT. Health Technol Assess. 2020;24(33):1-70. https:// doi.org/10.3310/hta24330

4. Mor G, Cardenas I. The immune system in pregnancy: a unique complexity, Am J Reprod Immunol (New York, NY: 1989). 2010;63(6):425-33.

5. Mor $G$, Aldo P, Alvero AB. The unique immunological and microbial aspects of pregnancy. Nat Rev Immunol. 2017;17(8):469-82. https://doi.org/10.1038/ nri.2017.64.

6. Michel MZ, Khong TY, Clark DA, Beard RW. A morphological and immunological study of human placental bed biopsies in miscarriage. Br J Obstet Gynaecol. 1990;97(11):984-8. https://doi.org/10.1111/j.1471-0528.1 990.tb02468.x

7. Giakoumelou S, Wheelhouse N, Brown J, Wade J, Simitsidellis I, Gibson D, et al. Chlamydia trachomatis infection of human endometrial stromal cells induces defective decidualisation and chemokine release. Sci Rep. 2017;7(1): 2001. https://doi.org/10.1038/s41598-017-02223-z.

8. Osman I, Young A, Ledingham MA, Thomson AJ, Jordan F, Greer IA, et al. Leukocyte density and pro-inflammatory cytokine expression in human fetal membranes, decidua, cervix and myometrium before and during labour at term. Mol Hum Reprod. 2003;9(1):41-5. https://doi.org/10.1093/molehr/ga g001.

9. Frazier T, Hogue CJR, Bonney EA, Yount KM, Pearce BD. Weathering the storm; a review of pre-pregnancy stress and risk of spontaneous abortion. Psychoneuroendocrinology. 2018:92:142-54. https://doi.org/10.1016/j. psyneuen.2018.03.001.

10. Qian J, Zhang N, Lin J, Wang C, Pan X, Chen L, et al. Distinct pattern of Th17/Treg cells in pregnant women with a history of unexplained recurrent spontaneous abortion. Biosci Trends. 2018;12(2):157-67. https://doi.org/10. 5582/bst.2018.01012

11. Aagaard K, Riehle K, Ma J, Segata N, Mistretta TA, Coarfa C, et al. A metagenomic approach to characterization of the vaginal microbiome signature in pregnancy. PLoS One. 2012;7(6):e36466. https://doi.org/10.1371/ journal.pone.0036466.

12. Maclntyre DA, Chandiramani M, Lee YS, Kindinger L, Smith A, Angelopoulos $\mathrm{N}$, et al. The vaginal microbiome during pregnancy and the postpartum period in a European population. Sci Rep. 2015;5(1):8988. https://doi.org/1 0.1038/srep08988

13. Spear GT, French AL, Gilbert D, Zariffard MR, Mirmonsef P, Sullivan TH, et al. Human alpha-amylase present in lower-genital-tract mucosal fluid processes glycogen to support vaginal colonization by Lactobacillus. J Infect Dis. 2014; 210(7):1019-28. https://doi.org/10.1093/infdis/jiu231.

14. Witkin SS, Linhares IM. Why do lactobacilli dominate the human vaginal microbiota? BJOG. 2017:124(4):606-11. https://doi.org/10.1111/1471-052 8.14390. 
15. Brown RG, Marchesi JR, Lee YS, Smith A, Lehne B, Kindinger LM, et al. Vaginal dysbiosis increases risk of preterm fetal membrane rupture, neonatal sepsis and is exacerbated by erythromycin. BMC Med. 2018;16(1):9. https:// doi.org/10.1186/s12916-017-0999-x.

16. Callahan BJ, DiGiulio DB, Goltsman DSA, Sun CL, Costello EK, Jeganathan P, et al. Replication and refinement of a vaginal microbial signature of preterm birth in two racially distinct cohorts of US women. Proc Natl Acad Sci U S A. 2017;114(37):9966-71. https://doi.org/10.1073/pnas.1705899114.

17. Kindinger LM, Bennett PR, Lee YS, Marchesi JR, Smith A, Cacciatore S, et al. The interaction between vaginal microbiota, cervical length, and vaginal progesterone treatment for preterm birth risk. Microbiome. 2017;5(1):6. https://doi.org/10.1186/s40168-016-0223-9.

18. Kindinger LM, Maclntyre DA, Lee YS, Marchesi JR, Smith A, McDonald JA, et al. Relationship between vaginal microbial dysbiosis, inflammation, and pregnancy outcomes in cervical cerclage. Sci Transl Med. 2016;8(350): 350ra102.

19. Taylor BD, Holzman CB, Fichorova RN, Tian Y, Jones NM, Fu W, et al. Inflammation biomarkers in vaginal fluid and preterm delivery. Hum Reprod (Oxford, England). 2013;28(4):942-52.

20. Brown RG, Al-Memar M, Marchesi JR, Lee YS, Smith A, Chan D, et al. Establishment of vaginal microbiota composition in early pregnancy and its association with subsequent preterm prelabor rupture of the fetal membranes. Transl Res. 2019;207:30-43. https://doi.org/10.1016/j.trsl.2018.12. 005.

21. Al-Memar M, Bobdiwala S, Fourie H, Mannino R, Lee YS, Smith A, et al. The association between vaginal bacterial composition and miscarriage: a nested case-control study. BJOG. 2020;127(2):264-74. https://doi.org/1 0.1111/1471-0528.15972.

22. Abdallah Y, Daemen A, Kirk E, Pexsters A, Naji O, Stalder C, et al. Limitations of current definitions of miscarriage using mean gestational sac diameter and crown-rump length measurements: a multicenter observational study. Ultrasound Obstet Gynecol. 2011;38(5):497-502. https://doi.org/10.1002/ uog.10109.

23. Preisler J, Kopeika J, Ismail L, Vathanan V, Farren J, Abdallah Y, et al. Defining safe criteria to diagnose miscarriage: prospective observational multicentre study. BMJ (Clin Res ed). 2015;351:h4579-h.

24. Bottomley C, Bourne T. Diagnosing miscarriage. Best Pract Res Clin Obstet Gynaecol. 2009;23(4):463-77. https://doi.org/10.1016/j.bpobgyn.2009.02.004

25. Bottomley C, Van Belle V, Mukri F, Kirk E, Van Huffel S, Timmerman D, et al. The optimal timing of an ultrasound scan to assess the location and viability of an early pregnancy. Hum Reprod (Oxford, England). 2009;24(8): 1811-7

26. Vialard F, Simoni G, Aboura A, De Toffol S, Molina Gomes D, Marcato L, et al. Prenatal BACs-on-Beads ${ }^{\mathrm{TM}}$ : a new technology for rapid detection of aneuploidies and microdeletions in prenatal diagnosis. Prenat Diagn. 2011; 31(5):500-8. https://doi.org/10.1002/pd.2727.

27. Allingham-Hawkins DJ, Chitayat D, Cirigliano V, Summers A, Tokunaga J, Winsor $E$, et al. Prospective validation of quantitative fluorescent polymerase chain reaction for rapid detection of common aneuploidies. Genet Med. 2011;13(2):140-7. https://doi.org/10.1097/GIM.0b013e3182036763.

28. Mclnnes PC M. Manual of Procedures - Human Microbiome Project Version 12.0 Core Microbiome Sampling Protocol A 29 Jul 2010 1-2 The Steering Committee has the responsibility for developing protocol amendments. https://www.ncbi.n/m.nih.gov/projects/gap/cgi-bin/GetPdf.cgi?id=phd0031 90.2. Accessed 13 Jan 2022

29. Kozich JJ, Westcott SL, Baxter NT, Highlander SK, Schloss PD. Development of a dual-index sequencing strategy and curation pipeline for analyzing amplicon sequence data on the MiSeq Illumina sequencing platform. Appl Environ Microbiol. 2013;79(17):5112-20. https://doi.org/10.1128/AEM.01 043-13.

30. Wang Q, Garrity GM, Tiedje JM, Cole JR. Naive Bayesian classifier for rapid assignment of rRNA sequences into the new bacterial taxonomy. Appl Environ Microbiol. 2007;73(16):5261-7. https://doi.org/10.1128/AEM.00062-07.

31. France MT, Ma B, Gajer P, Brown S, Humphrys MS, Holm JB, et al. VALENCIA: a nearest centroid classification method for vaginal microbial communities based on composition. Microbiome. 2020;8(1):166. https://doi.org/10.1186/s4 0168-020-00934-6.

32. Segata N, Izard J, Waldron L, Gevers D, Miropolsky L, Garrett WS, et al. Metagenomic biomarker discovery and explanation. Genome Biol. 2011; 12(6):R60. https://doi.org/10.1186/gb-2011-12-6-r60.
33. Schwager E, Mallick H, Ventz S, Huttenhower C. A Bayesian method for detecting pairwise associations in compositional data. PLoS Comput Biol. 2017;13(11):e1005852. https://doi.org/10.1371/journal.pcbi.1005852.

34. Epskamp SCA, Waldorp LJ, Schmittmann VD, Borsboom D. qgraph: network visualizations of relationships in psychometric data. J Stat Softw. 2012;48(4).

35. Bender Atik R, Christiansen OB, Elson J, Kolte AM, Lewis S, Middeldorp S, et al. ESHRE guideline: recurrent pregnancy loss. Hum Reprod Open. 2018; 2018(2):hoy004

36. RCOG. Green Top Guideline. The investigation and treatment of couples with recurrent first-trimester and second-trimester miscarriage. RCOG Green Top Guidel. 2011;17:1-17.

37. Jia CW, Wang L, Lan $Y L$, Song R, Zhou LY, Yu L, et al. Aneuploidy in early miscarriage and its related factors. Chin Med J. 2015;128(20):2772-6. https:// doi.org/10.4103/0366-6999.167352.

38. Choi TY, Lee HM, Park WK, Jeong SY, Moon HS. Spontaneous abortion and recurrent miscarriage: a comparison of cytogenetic diagnosis in 250 cases. Obstet Gynecol Sci. 2014;57(6):518-25. https://doi.org/10.5468/ogs.2014.57.6. 518.

39. Franasiak JM, Forman EJ, Hong KH, Werner MD, Upham KM, Treff NR, et al. The nature of aneuploidy with increasing age of the female partner: a review of 15,169 consecutive trophectoderm biopsies evaluated with comprehensive chromosomal screening. Fertil Steril. 2014;101(3):656-63.e1.

40. Teklenburg G, Salker M, Molokhia M, Lavery S, Trew G, Aojanepong T, et al. Natural selection of human embryos: decidualizing endometrial stromal cells serve as sensors of embryo quality upon implantation. PLoS One. 2010; 5(4):e10258. https://doi.org/10.1371/journal.pone.0010258.

41. Lucas ES, Dyer NP, Murakami K, Lee YH, Chan YW, Grimaldi G, et al. Loss of endometrial plasticity in recurrent pregnancy loss. Stem Cells. 2016;34(2): 346-56. https://doi.org/10.1002/stem.2222.

42. Lucas ES, Vrljicak P, Muter J, Diniz-da-Costa MM, Brighton PJ, Kong CS, et al. Recurrent pregnancy loss is associated with a pro-senescent decidual response during the peri-implantation window. Commun Biol. 2020;3(1):37. https://doi.org/10.1038/s42003-020-0763-1.

43. Ashford KB, Chavan N, Ebersole JL, Wiggins AT, Sharma S, McCubbin A, et al. Patterns of systemic and cervicovaginal fluid inflammatory cytokines throughout pregnancy. Am J Perinatol. 2018;35(5):455-62. https://doi.org/1 0.1055/s-0037-1608677.

44. Bayar E, Bennett PR, Chan D, Sykes L, Maclntyre DA. The pregnancy microbiome and preterm birth. Semin Immunopathol. 2020;42(4):487-99. https://doi.org/10.1007/s00281-020-00817-w.

45. Bennett PR, Brown RG, Maclntyre DA. Vaginal microbiome in preterm rupture of membranes. Obstet Gynecol Clin North Am. 2020;47(4):503-21. https://doi.org/10.1016/j.ogc.2020.08.001.

46. Chen H, Yang X, Du J, Lu M. Interleukin-18 gene polymorphisms and risk of recurrent pregnancy loss: a systematic review and meta-analysis. J Obstet Gynaecol Res. 2015;41(10):1506-13. https://doi.org/10.1111/jog.12800.

47. Jacobsson B, Holst RM, Mattsby-Baltzer I, Nikolaitchouk N, Wennerholm UB, Hagberg H. Interleukin-18 in cervical mucus and amniotic fluid: relationship to microbial invasion of the amniotic fluid, intra-amniotic inflammation and preterm delivery. BJOG. 2003;110(6):598-603. https://doi.org/10.1046/j.1471-0528.2003.02445.x.

48. Jespers V, Kyongo J, Joseph S, Hardy L, Cools P, Crucitti T, et al. A longitudinal analysis of the vaginal microbiota and vaginal immune mediators in women from sub-Saharan Africa. Sci Rep. 2017:7(1):11974. https://doi.org/10.1038/s41598-017-12198-6.

49. Masson L, Barnabas S, Deese J, Lennard K, Dabee S, Gamieldien H, et al. Inflammatory cytokine biomarkers of asymptomatic sexually transmitted infections and vaginal dysbiosis: a multicentre validation study. Sex Transm Infect. 2019;95(1):5-12. https://doi.org/10.1136/sextrans-2017-053506.

50. Castro J, Jefferson KK, Cerca N. Genetic heterogeneity and taxonomic diversity among Gardnerella species. Trends Microbiol. 2020;28(3):202-11. https://doi.org/10.1016/j.tim.2019.10.002.

51. Fettweis JM, Serrano MG, Brooks JP, Edwards DJ, Girerd PH, Parikh HI, et al. The vaginal microbiome and preterm birth. Nat Med. 2019;25(6):1012-21. https://doi.org/10.1038/s41591-019-0450-2.

52. Borgdorff H, Gautam R, Armstrong SD, Xia D, Ndayisaba GF, van Teijlingen $\mathrm{NH}$, et al. Cervicovaginal microbiome dysbiosis is associated with proteome changes related to alterations of the cervicovaginal mucosal barrier. Mucosal Immunol. 2016;9(3):621-33. https://doi.org/10.1038/mi.2015.86.

53. Anahtar MN, Byrne EH, Doherty KE, Bowman BA, Yamamoto HS, Soumillon $M$, et al. Cervicovaginal bacteria are a major modulator of host 
inflammatory responses in the female genital tract. Immunity. 2015;42(5): 965-76. https://doi.org/10.1016/j.immuni.2015.04.019.

54. Chen C, Song X, Wei W, Zhong H, Dai J, Lan Z, et al. The microbiota continuum along the female reproductive tract and its relation to uterinerelated diseases. Nat Commun. 2017;8(1):875. https://doi.org/10.1038/s414 67-017-00901-0.

55. Moreno I, Codoner FM, Vilella F, Valbuena D, Martinez-Blanch JF, JimenezAlmazan J, et al. Evidence that the endometrial microbiota has an effect on implantation success or failure. Am J Obstet Gynecol. 2016;215(6):684-703. https://doi.org/10.1016/j.ajog.2016.09.075.

56. Heng YJ, Liong S, Permezel M, Rice GE, Di Quinzio MK, Georgiou HM. Human cervicovaginal fluid biomarkers to predict term and preterm labor. Front Physiol. 2015;6:151. https://doi.org/10.3389/fphys.2015.00151.

57. Zegels G, Van Raemdonck GA, Tjalma WA, Van Ostade XW. Use of cervicovaginal fluid for the identification of biomarkers for pathologies of the female genital tract. Proteome Sci. 2010;8(1):63. https://doi.org/10.11 86/1477-5956-8-63

58. Lamont RF. The role of infection in preterm labour and birth. Hosp Med. 2003;64(11):644-7. https://doi.org/10.12968/hosp.2003.64.11.2343.

59. Lash GE, Ernerudh J. Decidual cytokines and pregnancy complications: focus on spontaneous miscarriage. J Reprod Immunol. 2015;108:83-9. https://doi. org/10.1016/j.ji.2015.02.003.

60. Naeimi S, Ghiam AF, Mojtahedi Z, Dehaghani AS, Amani D, Ghaderi A. Interleukin-18 gene promoter polymorphisms and recurrent spontaneous abortion. Eur J Obstet Gynecol Reprod Biol. 2006;128(1-2):5-9. https://doi. org/10.1016/j.jogrb.2006.02.012.

61. Wang ZC, Yunis EJ, De los Santos MJ, Xiao L, Anderson DJ, Hill JA. Thelper 1-type immunity to trophoblast antigens in women with a history of recurrent pregnancy loss is associated with polymorphism of the IL 1B promoter region. Genes Immun. 2002;3(1):38-42. https://doi.org/10.1038/sj. gene.6363812

62. Farren J, Jalmbrant M, Falconieri N, Mitchell-Jones N, Bobdiwala S, Al-Memar $\mathrm{M}$, et al. Posttraumatic stress, anxiety and depression following miscarriage and ectopic pregnancy: a multicenter, prospective, cohort study. Am J Obstet Gynecol. 2020;222(4):367.e1-e22

\section{Publisher's Note}

Springer Nature remains neutral with regard to jurisdictional claims in published maps and institutional affiliations.

Ready to submit your research? Choose BMC and benefit from:

- fast, convenient online submission

- thorough peer review by experienced researchers in your field

- rapid publication on acceptance

- support for research data, including large and complex data types

- gold Open Access which fosters wider collaboration and increased citations

- maximum visibility for your research: over $100 \mathrm{M}$ website views per year

At $\mathrm{BMC}$, research is always in progress.

Learn more biomedcentral.com/submissions 